\title{
Anticoagulación profiláctica en pacientes hospitalizados durante la COVID-19: menos es mejor
}

\section{Prophylactic anticoagulation in hospitalized patients with COVID-19: less is better}

\author{
Mateo Porres-Aguilar ${ }^{1 *}$, Orlando R. Pérez-Nieto², Javier E. Anaya-Ayala³, Eder I. Zamarrón-López \\ Thierry Hernández-Gilsoul y Moisés Aurón ${ }^{6}$ \\ ${ }^{1}$ Department of Internal Medicine, Division of Hospital Medicine, Texas Tech University Health Sciences Center, EI Paso, Texas, EE.UU.; \\ ${ }^{2}$ Departamento de Cuidados Intensivos y Medicina Critica, Hospital San Juan del Río, Qro., México; ${ }^{3}$ Departamento de Cirugía, Sección de Cirugía \\ Vascular y Terapia Endovascular; Instituto Nacional de Ciencias Médicas y Nutrición Salvador Zubirán, Ciudad de México, Méx., México; ${ }^{4}$ Unidad \\ de Cuidados Intensivos y Medicina Crítica, Hospital CEMAIN-Tampico y Hospital IMSS n. 6 , Ciudad Madero, Tamps., México; ${ }^{5}$ Departamento de \\ Medicina de Emergencias, Instituto Nacional de Ciencias Médicas y Nutrición Salvador Zubirán, Ciudad de México, Méx., México; ${ }^{6}$ Department of \\ Hospital Medicine and Pediatric Hospital Medicine, Cleveland Clinic, Cleveland, Ohio, EE.UU.
}

Leímos con interés el artículo publicado por IgnacioIbarra, et al.1. Los autores proponen anticoagulación terapéutica con heparinas y/o rivaroxabán para pacientes hospitalizados con enfermedad por coronavirus 2019 (COVID-19) sin mejoría clínica o con dímero D > 3,000 ng/ml. Queremos compartir lo siguiente: recientemente se publicó el estudio ACTION, que comparó tratamiento anticoagulante a dosis terapéuticas vs. profilácticas en 615 pacientes $^{2}$. El objetivo primario fue analizar jerárquicamente la mortalidad, tiempo hospitalario y suplemento de oxígeno a 30 días. El 90\% de los pacientes con estrategia de anticoagulación terapéutica recibió rivaroxabán $20 \mathrm{mg}$ vía oral al día. No se encontraron diferencias significativas entre ambos grupos en cuanto al objetivo primario compuesto: estrategia terapéutica $34.8 \%$ vs. estrategia profiláctica $41.3 \%$ (odds ratio [OR]: 0.86; intervalo de confianza del 95\% [IC 95\%]: 0.59-1.22; $p=0.40$ ). Sin embargo, existió diferencia significativa entre ambos grupos relacionada con los objetivos de seguridad, hemorragia mayor o clínicamente relevante: estrategia terapéutica $8 \%$ vs. estrategia profiláctica $2 \%$ (OR: 3.6; IC 95\%: 1.61-8.27, $p=0.001)^{2}$. Estos resultados muestran que una estrategia de anticoagulación con dosis terapéutica no confirió beneficio en comparación a una estrategia de tromboprofilaxis convencional ${ }^{2}$.

Consideramos que se debe mantener cautela en las estrategias de tromboprofilaxis COVID-19 debido al alto riesgo de eventos adversos, el cual puede superar su beneficio en el contexto de escenarios clínicos difíciles. Se sugiere evitar dichas estrategias basadas solamente en niveles elevados de dímero $\mathrm{D}$ o falta de mejoría clínica. La anticoagulación terapéutica permanece reservada para eventos trombóticos agudos debidamente diagnosticados. Próximas publicaciones de resultados de ensayos multiplataforma, adaptativos, prospectivos, aleatorizados (REMA-CAP, ATTACC y ACTIV-4a) brindarán evidencia más sólida sobre estrategias tromboprofilácticas para estos pacientes ${ }^{3}$.

\section{Financiamiento}

Los autores declaran no haber recibido financiamiento

\section{Conflicto de intereses}

Los autores declaran no tener conflicto de intereses.

\section{Bibliografía}

1. Ignacio-Ibarra G, García-Lee MT, González-Ávila Al, García-Chávez J, Guzmán-Chores L, Cruz CL, et al. Propuesta para el manejo de la coagulopatía asociada a COVID-19 en adultos. Gac Med Mex. 2021:157:209-14.

2. Lopes RD, de Barros e Silva PGM, Furtado RHM, Macedo AVS, Bronhara B, Damiani LP, et al. Therapeutic versus prophylactic anticoagulation for patients admitted to hospital with COVID-19 and elevated D-dimer concentration (ACTION): an open-label, multicentre, randomised, controlled trial. Lancet. 2021;397:2253-63.

3. Porres-Aguilar M, Pérez-Nieto OR, Zamarrón-López Él, Namendys-Silva SA. Thromboprophylaxis strategies for COVID-19 patients. Arch Med Res. 2021; Jul 5: S0188-4409(21)00146-6. doi: 10.1016/j.arcmed.2021.06.011. Online ahead of print.
Correspondencia:

*Mateo Porres-Aguilar

E-mail: mporres1980@gmail.com

0016-3813/@ 2021 Academia Nacional de Medicina de México, A.C. Publicado por Permanyer.

CC BY-NC-ND (http://creativecommons.org/licenses/by-nc-nd/4.0/)
Gac Med Mex. 2021;157:661

Disponible en PubMed

www.gacetamedicademexico.com 